\title{
Planning and Execution of Access for Percutaneous Renal Stone Removal in a Community Hospital Setting
}

\author{
Robert M. Springer III, MD ${ }^{1}$ \\ ${ }^{1}$ Department of Radiology, Lancaster Regional Medical Center, \\ Lancaster, Pennsylvania \\ Semin Intervent Radiol 2015;32:311-322
}

Address for correspondence Robert M. Springer III, MD, Department of Radiology, Lancaster Regional Medical Center, 250 College Avenue, Lancaster, PA 17603 (e-mail: rmspringer3@verizon.net).
There have been several reports on the feasibility and safety of the superior pole approach to the kidney for percutaneous renal stone removal. To the author's knowledge, no one has published on the huge advantages this approach affords, owing to the rigid nature of the nephroscope/ultrasonic lithotripter, and due to the usually oblique position of the kidney as it lies upon the psoas. Between July 1986 and June 2014, the author has performed more than 500 percutaneous renal access procedures for stone removal at a single community hospital. Procedural technique is reviewed in detail, including the usual supremacy of the superior pole approach, the value of preprocedural sagittal computed tomographic reconstructions in forming a tentative plan of approach to the kidney, and the value of an intraprocedural partial-air nephrostogram in finalizing the plan. The importance of collaboration with the involved urologist, and knowledge of the tools available to the urologist, is emphasized.

In the first half of the 1980s, with the introduction of extracorporeal shock-wave lithotripsy and percutaneous nephrolithotomy, there was an abrupt advance in the treatment of renal calculi. Prior to that time the only option for treatment of large renal calculi, particularly staghorns, had been surgery. For these stones, a combined percutaneousextracorporeal technique quickly evolved. ${ }^{1}$

The author's first exposure to these techniques was in 1985, at the University of Virginia in Charlottesville. Here two or three "staghorn percs" were performed each week. These cases were very much team efforts; interventional radiologists created the initial access tract or tracts, and then the stone was addressed by a urologist. Decision making on subsequent maneuvers was collaborative.

Since 1986, in a single community hospital private practice setting, these methods have continued to serve well. Over the years, in a personal series of more than 500 cases, a few modifications have been made. Below are practical and technical points intended to help anyone starting out doing these procedures. ${ }^{2,3}$

The team approach has proven successful and efficient in the author's community hospital setting, as it makes best use of the skill and time of the radiologist, urologist, and anesthesiologist, and also of the available equipment. Procedures are staged such that the access is created in the morning, in the Department of Radiology's Special Procedure suite. Conscious sedation is used for the access procedure. The patient is then returned to the outpatient surgery nursing unit until the afternoon, when he or she is taken to the operating room (OR), where general anesthesia is induced, the tract dilated, and the stone removed. Proceeding this way we take advantage of the superb fluoroscopy available in the Department of Radiology (as opposed to the mobile C-arms used in the ORs), and the urologist and anesthesiologist need not waste time while the percutaneous access is being obtained.

\section{The Urologist's Armamentarium}

The task of the interventional radiologist is not (usually) to remove the stone from the kidney; rather it is to provide safe and effective access for the urologist to do that. "Safe" is one thing, "effective" is another. To plan an effective access, it is very helpful to understand some of the capabilities and limitations of the tools the urology colleague has at their disposal.

By far the most generally useful tools in dealing with larger stone burdens are the rigid nephroscope and ultrasonic lithotripter. Various grasping instruments can be also used to good effect through the nephroscope. The scopes we use fit through a 26-Fr sheath, though there is surprisingly no increase in complications using a 30-Fr sheath, and the larger sheath will sometimes allow extraction of a big stone intact.

The huge limitation of these instruments is their rigidity, and it is this single fact that governs much of the decision
Issue Theme Endovascular Aortic Aneurysm Repair; Guest Editor, S. William Stavropoulos, MD, FSIR
Copyright $\odot 2015$ by Thieme Medical Publishers, Inc., 333 Seventh Avenue, New York, NY 10001, USA. Tel: +1(212) 584-4662.
DOI http://dx.doi.org/ 10.1055/s-0035-1556880. ISSN 0739-9529. 


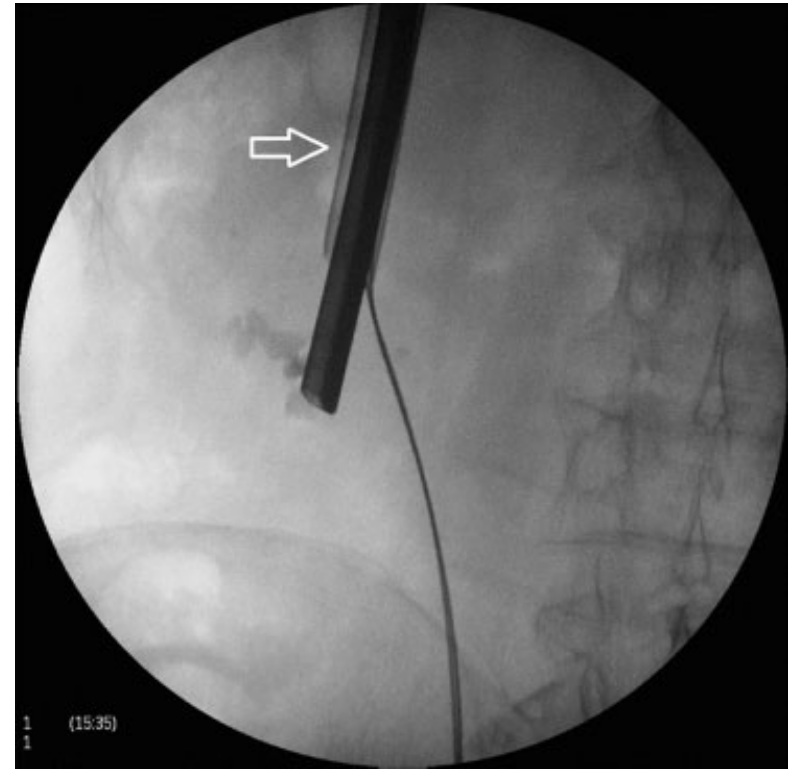

Fig. 1 Rigid nephroscope (arrow) engaging a renal stone; obviously, this instrument will not bend.

making involved in the radiologist's approach to the kidney (-Fig. 1). This will be discussed in detail later.

Today, the urologist has more flexible tools at hand. The newer cystoscopes are so flexible that they can turn back upon themselves; they are invaluable in reaching stones in isolated calyces, especially when the infundibula are long and narrow (-Fig. 2).

However, even when the urologist is able to maneuver a flexible scope so as to reach a stone, the procedure may not be over. Laser fibers used in lithotripsy are stiff, so they will not necessarily be able to make a bend as tight as the diagnostic scope will. Baskets will make almost any bend a scope will, but if there is a larger stone in a calyx at the end of a tight infundibulum, removing the stone with a basket may cause significant traumatic injury.

A flexible ureteroscope is often used to examine the ureter toward the end of the case. Baskets advanced through this type of scope are used to remove larger stone fragments that have entered the ureter, although sometimes the primary target is a ureteral stone. Again, a straighter approach may allow a larger laser fiber to be used, shortening procedure time and earning your urologist's appreciation.

\section{The Position of the Kidney upon the Psoas}

With the idea firmly in mind that the urologists' tools are not all that flexible, the orientation of the kidney as it rides on the psoas muscle should be considered.

If the reader has been in practice for 10 years or so, he or she will remember the days when the standard presentation of a computed tomographic (CT) scan was a series of transverse (the " $\mathrm{A}$ " for axial in the former term "CAT") images. At some institutions, the radiologist could request

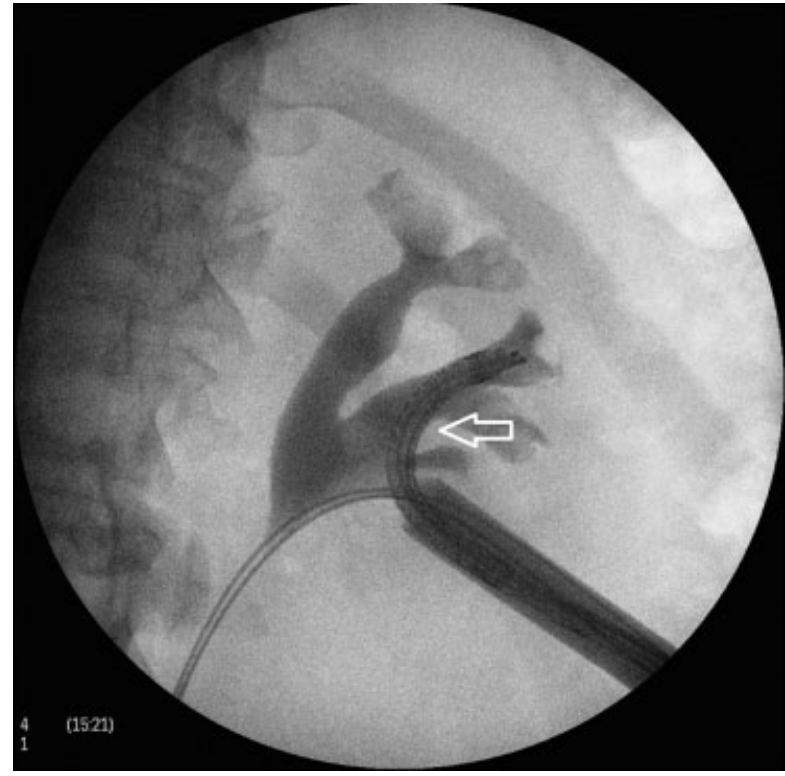

Fig. 2 Flexible cystoscope in an upper pole calyx (arrow). More of a bend then this, and a large laser fiber might be too stiff to be advanced through bend in the scope.

reconstructions in coronal, sagittal, and off-standard planes.

When all that was available were transverse images, it was hard to appreciate just how variable the longitudinal plane of the kidney can be. In 2015, however, many CTs are presented on workstations with coronal and sagittal recons readymade. The sagittal reconstructions are particularly revealing. If you examine several of them, with an eye to the kidney's position, you will see that its long axis varies from almost straight "up and down" to almost transverse, with the superior pole posteriorly directed.

The orientation of the kidney upon the psoas is hugely important to the optimum percutaneous approach. Imagine, for example, making a puncture of an inferior pole calyx in a nearly horizontal kidney. Although this will permit calyceal access, this will have placed the access into the "deepest" part of the kidney. As attempts to remove more and more of the stones are made, the nephroscope will have to be angled back and progressively more superficially. In a virgin retroperitoneum, the kidney has some mobility and may tilt a little, but the scope is very rigid and may potentially place very significant torque on the kidney. If the operator is working on a staghorn in such a kidney, he or she will be lucky if the lower pole and pelvis can be cleared of stones with this approach.

Now imagine approaching a superior pole calyx of this same horizontally lying kidney. After the urologist clears the superior pole of stones, the renal pelvis lies deeper, along the line of the scope. After the pelvis is cleared, the lower pole lies ahead, still deeper, again along the line of the scope, or nearly so. This superior pole approach therefore allows progressive advancement of the scope with clearing of the entire pelvicalyceal system (-Figs. 3 and $\mathbf{4}$ ). 


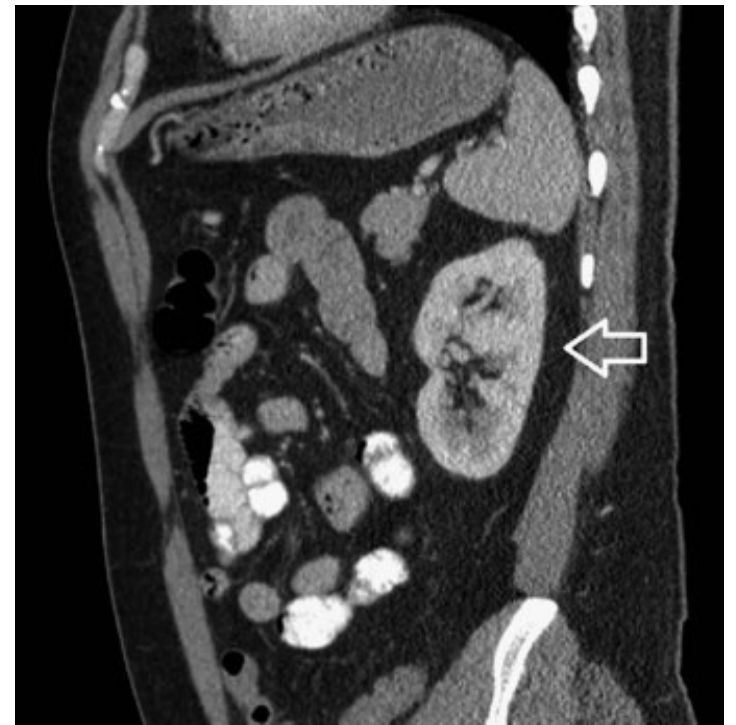

Fig. 3 Sagittal computed tomographic scan. As shown in this image, if the axis of the target kidney (arrow) is more or less vertical, there may not be a significant advantage to a superior pole approach.

\section{Approaching between the 11th and 12th Ribs}

As stated earlier, assuming the kidney is tilted more than minimally along the psoas, the most effective approach is often through a superior pole calyx ${ }^{4-8}(-$ Fig. 5). Sometimes it is possible to stay inferior to the ribs, but more often than not it is necessary to puncture between the 11th and 12th ribs. Before making the puncture, it is beneficial to mark the spot on the skin and check it fluoroscopically; the C-arm can be rotated into a lateral position to see the inferior tip of the lung in relation to the proposed puncture site. The same information can now be gained with add-on software that gives C-arms CT-type imaging capabilities, but rotating the C-arm is quicker with less radiation exposure.

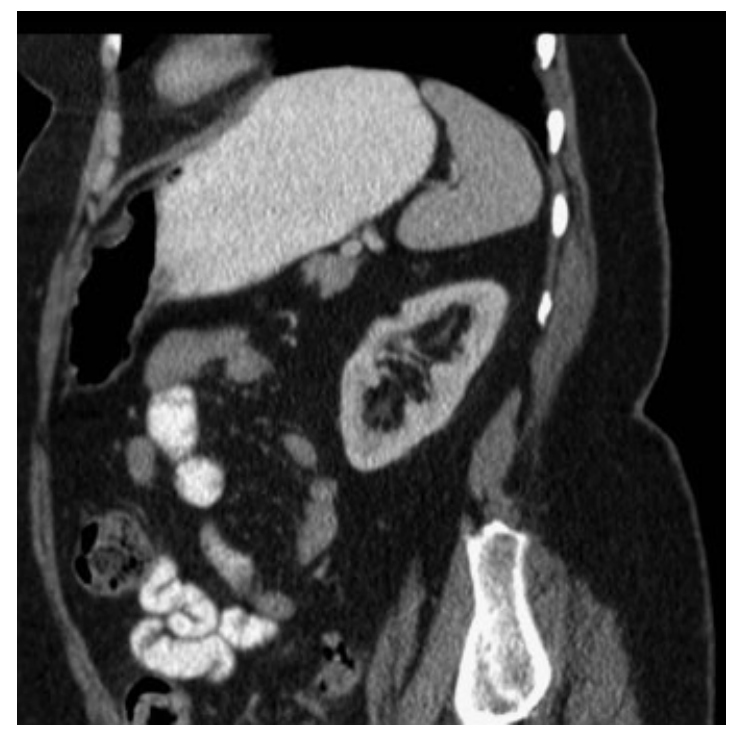

Fig. 4 Sagittal computed tomographic scan demonstrating a more typical kidney-axis, favoring a superior pole approach.
When puncturing between the 11th and 12th ribs, it is important to stay as close as possible to the 12th rib so as to avoid injury to the subcostal artery. You should assume your tract will pass through the inferior pleural reflection; the risks associated with violating the pleural space are acceptable, but the tract should not be dilated to $30-F r$ tract through the tip of the lung. The pleural reflection extends most inferiorly toward the midline; therefore, the further lateral the puncture is made, the greater the chance the pleural space will not be transgressed.

What are the risks of transgression of the pleural space? The most common problem is that of irrigant fluid leaking into the pleural space during urologic stone manipulation. Proper sheath placement (with the tip of the sheath in the collecting system) will, for the most part, prevent such leakage, but it can easily occur if the sheath retracts while the urologist is working on a stone in the superior calyceal system. A pneumothorax may also occur, but if the lung itself was not transgressed, the pneumothorax will be ex vacuo and self-limiting. Prior to the procedure, the approach should be explained to the anesthesiologist, so that he or she can alert you if there is a problem with oxygenation during the procedure. If the patient does desaturate, the C-arm can be repositioned over the chest to evaluate for the presence of a pneumothorax. The author has not encountered hemothorax, nephropleural fistula, or empyema. The first two of these complications have been reported in the literature, and the last is certainly possible. ${ }^{9}$

\section{Start With a Nephrostogram}

In some centers, an injectable retrograde ureteral catheter is routinely placed by the urology service as a preliminary procedure. My experience includes a few cases in which, for

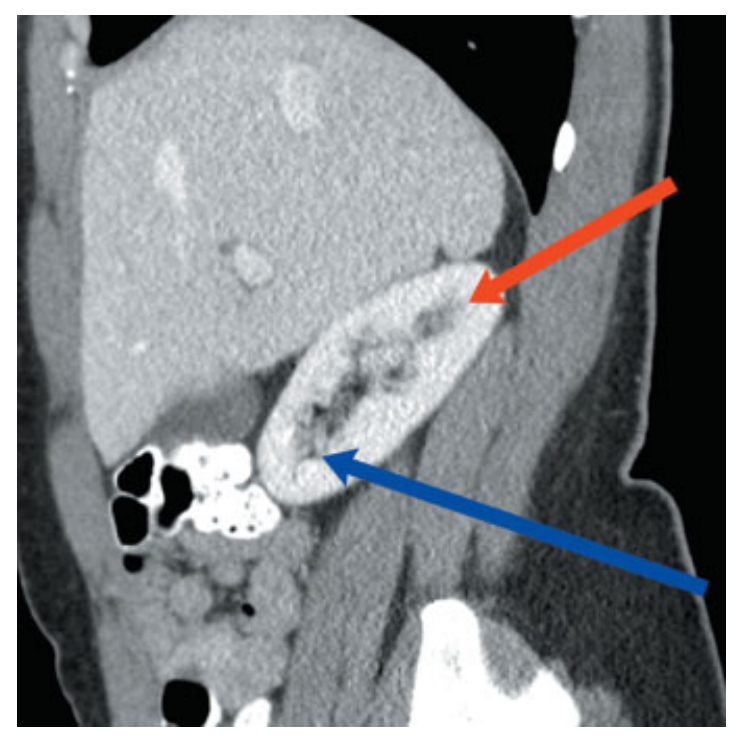

Fig. 5 In this example, the target kidney's axis is more than 45 degrees off the body's coronal plane. If the approach is via the inferior pole of this kidney (blue arrow), the urologist's ability to maneuver the rigid nephroscope will be limited. If a superior pole calyx is chosen for the approach (red arrow), most of the collecting system will be laid out in front of his scope. 
one reason or another, such a catheter was already in position. If one is present it can be helpful, but except in very unusual cases this step is not typically necessary.

Preprocedure evaluation of prior imaging (CT or intravenous pyelogram) is essential. CT is far preferable, as it will help assess the kidney's angle; determine the presence of renal cyst; and alert the operator to unusual positions of the colon, spleen, or liver.

Procedures are done with the patient in the prone or semiprone position. Be aware that with the patient prone, the kidney's position is often more superior (cephalad) than it appears on a supine CT. So a planned superior pole approach must sometimes be aborted, as it would require entering superior to the 11th rib and significantly increase the risk of lung injury. In most such cases, there will be a posteriorly directed middle or inferior pole calyx that should provide satisfactory access.

For the nephrostogram, the C-arm can be rotated somewhat laterally to avoid the paraspinal musculature as much as possible, and the needle can be lined up with a large stone. A stone in the pelvis or the superior loop of a double J ureteral stent also provides an excellent fluoroscopic target. The most foolproof approach is to center the needle and stone in the fluoroscopic field of view, with the needle foreshortened completely so that it shows up essentially as a dot or "bulls-eye." If this is done, parallax is not a concern, and all that must be done is to advance the needle on a straight path until the stone is struck.

After the stone is contacted, a small amount of contrast is injected. It is critical not to inject too much contrast, only to find that the needle tip is not in the collecting system, which may lead to contrast obstructing visualization of the stone or collecting system. For this step, the system's highest magnification factor and $\mathrm{R}$ level should be used. What should be seen is contrast flowing along the stone surface, into a part of the collecting system that is not filled with stone. If instead injection is performed outside of the collecting system, a pool of contrast will obscure at least a part of the target stone. This situation can usually be salvaged by targeting a different part of the stone or a different stone (assuming there is one). If that does not work, 50 to $100 \mathrm{~mL}$ of intravenous contrast can be administered; after the collecting system fills with contrast, a second target can be chosen.

If the collecting system is dilated, ultrasound can be used for the collecting system puncture. When the system is dilated, an "exchange opacification" technique should be performed. In this technique, no more contrast should be injected than urine aspirated, insofar as is practical. The goal is that the exchange technique will not increase the pressure in the collecting system, and thus not drive endotoxins from a colonized system into the bloodstream or cause a pressureinduced calyceal rupture.

Once collecting system access is obtained and 5 to $10 \mathrm{~mL}$ of contrast is injected, 5 to $6 \mathrm{~mL}$ of room air should also be injected. One must be careful not to inject too much air, as the endpoint should be to fill only about half the collecting system (-Fig. 6). This should be performed under fluoroscopic visualization, to be very sure the air fills the collecting system rather than vascular structures. A small air embolus might do no harm, but a paradoxical embolus through a patent foramen ovale using this technique has been reported. ${ }^{10}$ With the patient prone, the air will accumulate in the nondependent posteriorly directed calyces. These are the calyces closest to the posterior approach, and are the easiest and safest to puncture; through this approach, the tract will be established.

To minimize complications and to maximize success rates, the tract must always enter the collecting system through a calyx (preferably a fornix), or at the very least via a minor infundibulum. If the tract enters the collecting system more centrally, it may transgress larger vessels, increasing the chance of procedural and postprocedural bleeding. Also, if a tract is too central, it may inadvertently bypass stone peripheral to the entry point, where it may be very difficult to remove endoscopically.

\section{Study the Collecting System's Architecture}

It is not always necessary to puncture a superior pole calyx to gain most of the advantages of that approach. If a stone is confined to the inferior pole, a posteriorly directed inferior pole calyceal approach may also work. Such a calyx can be identified by rotating the C-arm (-Fig. 7).

Long slender infundibula are challenging, and duplicated and partially duplicated systems also create problems. The easiest architecture with which to work is a collecting system with short, broad infundibula, which facilitate instrument access (-Fig. 8).

If multiple stones located in calyces at the end of long, narrow infundibula are encountered, it may be impossible to access all the stones from any single approach. However, from a superior pole approach, a stone in the calyx punctured

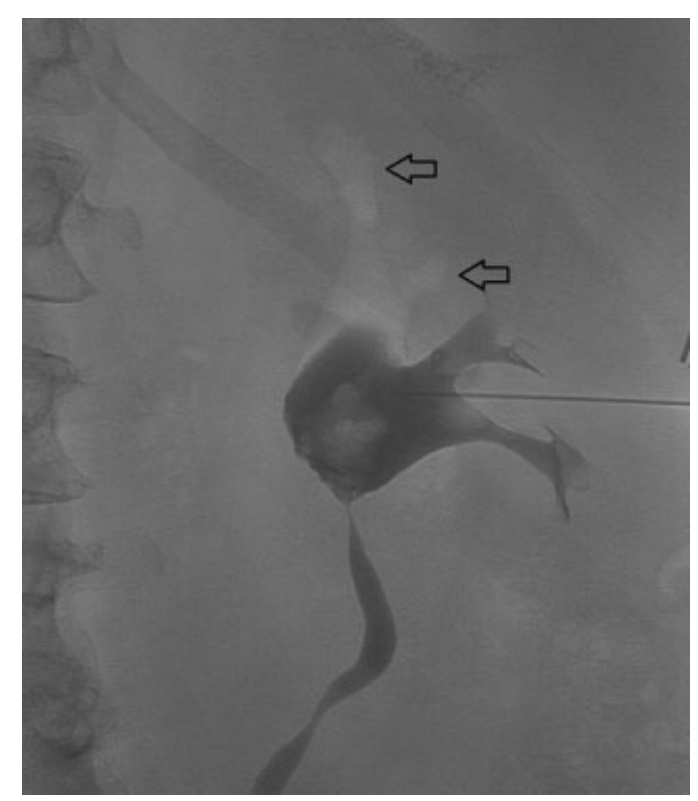

Fig. 6 Partial-air nephrostogram. With the patient prone, injected air will float to the nondependent posterior calyces (arrows), which will be closest to the skin. Sometimes the superior pole calyces may lie superior to the 11 th rib, but an air-filled (and thus posteriorly directed) middle pole calyx may also be targeted. 
a
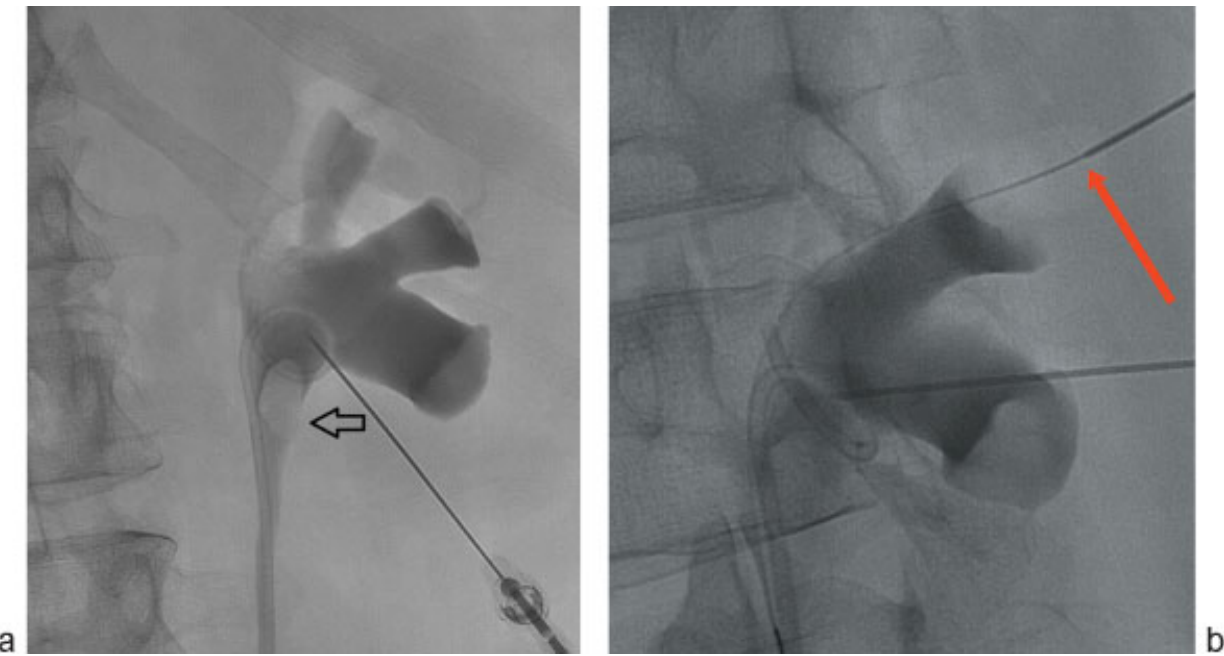

Fig. 7 (a) Nephrostogram demonstrates a stone in the proximal ureter (arrow) and the most inferior calyx. In this projection, it is difficult to visualize a posteriorly directed mid- or inferior pole calyx. (b) Same patient as in (a). As demonstrated on this oblique view, this air-filled calyx is superimposed upon a more antero inferior pole calyx on the frontal projection in (a). Note the point at which the wire exits the AccuStick needle (red arrow).

(if there is one), the minor and major infundibula, the renal pelvis, the major infundibulum to the inferior pole, and in any number of inferior pole infundibula and calyces can almost always be retrieved. The only stones that may not be able to be reached may be stones in nonpunctured superior pole calyces, and stones in middle pole calyces. To treat these, there are generally three options: prophylactically create another access (or accesses) in radiology; create additional access in the OR if it turns out to be necessary; or rely on extracorporeal lithotripsy to treat stones that cannot be reached. I tend to err on the side of creating a second access in radiology, where the fluoroscopic quality is better. Once an initial catheter is placed into the collecting system, the second puncture tends to go quickly due to the ability to inject contrast and/or air into the collecting system. There- fore, even if the second approach turns out to be unnecessary, only a little time has been lost.

When the collecting system is fully duplicated, each moiety must be treated based on its own architecture. A partially duplicated system can be difficult to approach via the superior pole: if the stone is in the inferior moiety, and the two moieties join too far medially, the rigid scope may not be able to be placed laterally into the inferior pole from the (usually small) pelvis. In this case, look for an air-filled calyx of the inferior moiety; this will be your best access.

\section{Puncturing an Empty Calyx}

Once the target calyx is decided upon, an approach must be planned. It is undesirable to approach a superior pole calyx

a
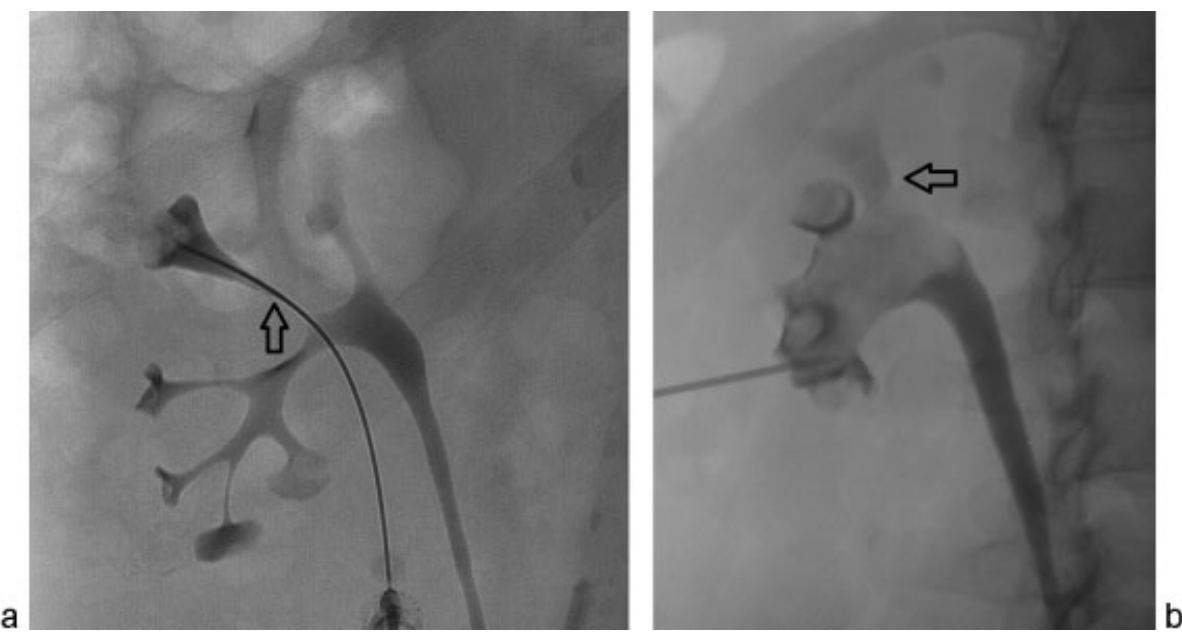

Fig. 8 (a) Nephrostogram demonstrating a long, narrow infundibulum (arrow). The rigid nephroscope's mobility is severely limited in such a system. (b) In contrast to (a) this nephrostogram demonstrates a short, broad infundibulum (arrow). It is much easier to work inside a system like this. 
from an inferior position-this defeats the whole idea of working with, not against, the rigid nephroscope. In general, it is best to approach a target calyx in the same plane as its infundibulum, as much as this is practical.

For a superior pole calyx, it may be helpful to angle the image intensifier a few degrees superiorly and a few degrees laterally, intending by doing this is to line up an approach staying inferior to the ribs. If this cannot be accomplished, the image intensifier (II) must be angled until an approach that clears the 12 th rib is identified ( $\mathbf{- F i g . 9}$ ). Once this approach is confirmed, the tip of a hemostat can be placed on the percutaneous access site and the II rotated to a horizontal position.

The author's preference for needle access is using the AccuStick system (Boston Scientific, Natick, MA), lining up the 21-gauge needle as for the nephrostogram so that the needle is seen directly on end. After the appropriate course is chosen, it is merely a matter of advancing the needle in a straight line. If the needle tends to deflect one way or another, it may help to rotate the bevel of the needle to the direction of the deflection. As the tip of the needle nears the calyx, the C-arm can be rotated as closely as practical to 90 degrees from the puncture angle. Air in the calyx can be seen to distort a little as the needle enters the calyx.

When the stylet is removed from the needle, bubbles of air from the calyx typically exit the needle hub. The 0.021-in. wire that comes with the AccuStick kit can now be advanced; it may help place a slight curve on the wire tip to give some directionality to the wire.

It is usually possible to maneuver the wire down the ureter. If not, the wire tip may be advanced into a mid- or lower-pole calyx, or coiled in the pelvis. The coaxial dilator and stiffener should be advanced together. Once the dilator is in the collecting system, the stiffener is removed and a TouhyBorst Y-connector can be placed over the wire to the hub of

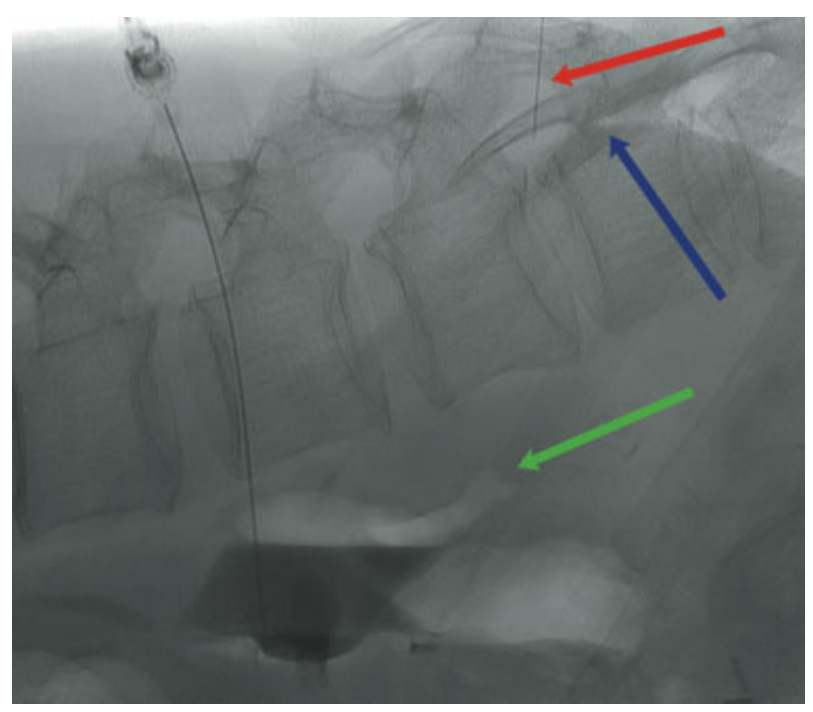

Fig. 9 Near lateral projection of the fluoroscopic beam shows that the projected puncture tract (as shown by 25-gauge anesthesia needlered arrow) avoids the tip of the lung (blue arrow). The target calyx is shown by green arrow. the dilator. The dilator can be withdrawn over the wire while injecting contrast until the dilator tip is in the parenchyma just outside the collecting system. The contrast syringe can be exchanged for one with Lidocaine, which is injected while pulling the dilator back along the tract to the skin. This ensures the best tract anesthesia possible. ${ }^{11}$

The AccuStick systems 6-Fr sheath is not usually needed. It is almost always possible to advance a standard 4-Fr dilator (tapered to advance over a 0.035 -in. wire) over the 0.021 -in AccuStick wire. Rotating the dilator continuously between the forefinger and the thumb helps advance it. Once the dilator tip is in the proximal ureter, the 0.021-in. wire is removed in favor of a 0.035-in. wire; the author typically uses a Benson wire (Cook Medical, Inc., Bloomington, IN). The wire is advanced to the bladder, and the dilator exchanged for a long 4-Fr catheter, such as a multiside hole straight flush catheter. Placing the catheter distally into the bladder decreases the likelihood of inadvertent removal of the catheter. The author secures the catheter with gauze and tape; placing a suture is typically unnecessary.

Avoiding several technical pitfalls is vital to postprocedural patient comfort. The intercostal approach is typically uncomfortable for the patient. Even the small difference in size between a 4- and a 5-Fr catheter can make a difference in patient tolerance, as does using the tract anesthesia technique. Finally, advancing too much of the catheter into the bladder can cause discomfort, and positioning the catheter tip in the very distal ureter is best.

\section{Puncturing a Stone-Filled Calyx}

Puncturing a stone-filled calyx can be a challenge. For stonefilled calyces, the author routinely uses with a hydrophilic angled Terumo (Terumo Medical Corporation, Somerset, NJ) guidewire to access the pelvocalyceal system. Sometimes, a 21-gauge needle and a 0.021-in. angled-tip Terumo are adequate; however, the more the stone to negotiate, the more likely the author will use an 18-gauge needle and a 0.035-in. Terumo. After engaging the stone with the needle, the tip is withdrawn very slightly and the wire advanced extremely gently. Again, the highest quality fluoroscopy should be used. It is very easy to perforate the uroepithelium with the wire and inadvertently exit the collecting system. Once that is done, the wire will tend preferentially to find the hole that was created; if this occurs repeatedly, the needle should be redirected to a different part of the target calyx. Also, increased space in the calyx can be created injecting contrast or saline through the nephrostogram needle, potentially facilitating wire advancement.

Once the needle has been advanced along the stone a few centimeters, a 4- or 5-Fr dilator can be advanced over the wire to help maintain purchase and forward progress along the stone. To be certain the dilator can be advanced without buckling in the soft tissues along the tract, a generous length of a 0.021 -in. wire should be advanced along the stone.

Once the wire is advanced beyond the stone and into the ureter, a stiffer wire should be exchanged for the initial access wire; the author prefers a Coons (Cook Medical, Inc., 
Bloomington, IN) or Amplatz (Cook Medical, Inc., Bloomington, IN) wire. If there is too much friction (again rotating the dilator may help) to advance a standard dilator along the stone, it may be necessary to use a hydrophilic catheter ( $\mathbf{F i g . ~ 1 0 ) . ~ I f ~ i t ~ i s ~}$ necessary to use a hydrophilic catheter to get down the ureter, this should be sutured in place, as it is too easily pulled out inadvertently.

Often, when dealing with a staghorn calculus, the calyces will be dilated but free of stone. In such a case, after entering a calyx with the needle, it is helpful to advance the needle through the empty calyx to a point along the inferior or superior edge of the most peripheral extent of the stone, before advancing the wire. The advantages of this maneuver are twofold. First, if the needle tip is not advanced to the stone, the wire will simply curl up in the dilated calyx. Although occasionally after a few coils, the wire will start to travel along the stone, this occurs relatively rarely. Second, placing the needle along the inferior or superior edge of the stone allows visualization of the wire to determine if it is traveling centrally in the narrow urine space along the stone. If the broad peripheral surface of the stone is initially engaged, it is difficult to determine whether the wire is in the urine space or if it has perforated the uroepithelium.

When starting to work a wire in a tight urine space along a stone, useful forward progress can be difficult without applying too much forward pressure. In this instance, the wire should be retracted, redirected, and another attempt made. Occasionally, especially when a hydrophilic wire makes an abrupt turn after exiting the needle tip, the wire may appear to be stuck when attempts to withdraw it are made. When this happens, forceful retraction of the wire is contraindicated because the needle tip will strip off the wire's hydrophilic coating. Owing to this potential complication, even with- drawing the needle and wire together completely out of the body is preferable to stripping off the wire's coating. In order not to lose needle position, it may help free the wire by advancing a Touhy adapter over the wire to the needle hub and lubricating the wire by injecting saline.

\section{Ureteral Stones}

With today's ureteroscopic equipment, it has become more uncommon for urologists to consult interventional radiologists to obtain a percutaneous approach to a ureteral stone. This referral may occur, for instance, when a very large prostate makes retrograde access to the ureter impossible. A superior pole approach, if feasible (staying inferior to the 11 th rib), is most helpful to the urologist. With this approach, in the past a rigid ureteroscope could often be advanced to the level of the midureter. The optics in flexible ureteroscopes have improved to the point where rigid ureteroscopes are rarely used, but from a superior approach even the flexible ureteroscope seems almost to fall down the ureter of its own accord. If the ureter proximal to the stone is dilated, it is often possible to address a ureteral stone with a flexible cystoscope, and sometimes even the rigid nephroscope and ultrasonic lithotripter can be used by this approach.

With diligence, it is almost always possible to maneuver a wire past a ureteral stone. Again an angled-tip Terumo wire is the author's first choice. Once the wire is advanced past the stone, there is often too much friction to advance anything other than a hydrophilic catheter over the wire to the bladder (-Fig. 11). If it can be done, it is very desirable to establish catheter position in the distal ureter. Once this is established, safety wire access should always be maintained; it is not too uncommon that the ureter is injured
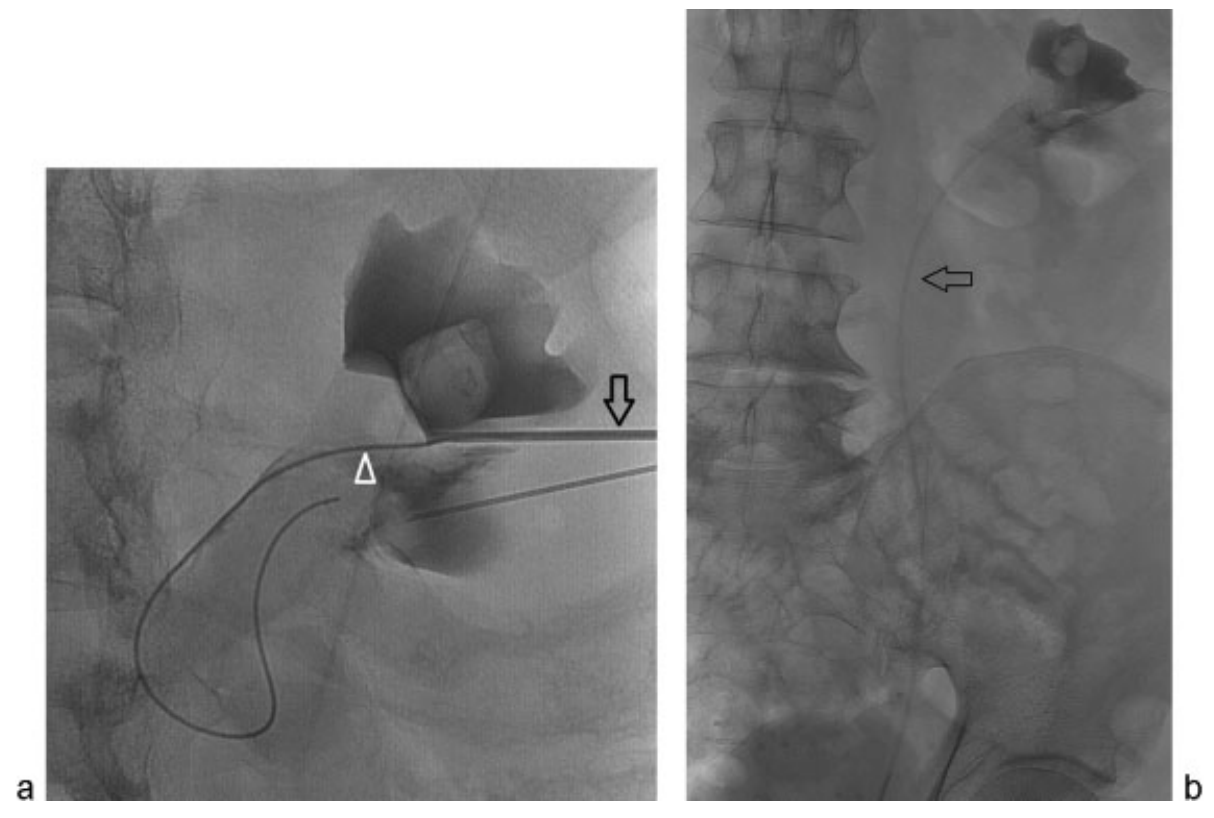

Fig. 10 In this patient, a staghorn calculus fills most of collecting system. (a) An 18-Gauge needle has been advanced to the surface of the stone in the superior calyx (black arrow). Through it, a hydrophilic wire has been advanced along the stone (white arrowhead). (b) This image shows final position of a 4-Fr catheter (arrow), with its tip in the distal ureter. 
enough in the process of removing a large impacted stone that it is hard to be sure of the true lumen afterward. If wire access to the bladder is maintained throughout the case, the operator remains in control; a double J stent can be advanced over the wire with perfect assurance that the stent lies in the ureter's true lumen.

\section{Calyceal Diverticula}

Occasionally, a patient will present with symptoms thought to be caused by a stone or stones in a calyceal diverticulum, and access to the diverticulum is requested (-Fig. 12). These cases are very delicate. ${ }^{12,13}$ The author's usual approach has been to start with a nephrostogram, so as to opacify the diverticulum and collecting system. However the diverticulum is targeted, an 18-gauge needle is used for puncture, because the needle has some directability once the tip is in the diverticulum and because of its stiffness it will not buckle in the tract. It often can be very difficult to identify the communication between the diverticulum and its calyx, and the wire will tend to curl up in the diverticulum. The operator must be very patient, trying minutely different angles with the needle and the wire tip. Sometimes the communication is so narrow that it is necessary to use a 0.021 -in. wire through the 18-gauge needle; again the angled-tip Terumo wires are the author's choice.

It is usually only possible to opacify the communication between the diverticulum and the collecting system very briefly-it is extremely helpful if such an image can be saved on a secondary monitor for reference. Subtraction fluoroscopy is usually not helpful because of the kidneys' motion with breathing. After advancing a wire through the communication and into the collecting system, a 4or $5-\mathrm{Fr}$ catheter is advanced to the bladder as described earlier.

\section{Dilating the Tract}

Except for rare cases in which general anesthesia is necessary for the initial nephrostogram, nephrostomy, and ureteral catheter placement, these initial access procedures are performed in the morning in the Department of Radiology's Interventional Radiology suite. Tract dilation is performed later, in the OR. Radiology provides an IR technician and a general radiology technician to the OR. The IR technician helps with positioning, prepping, draping, and assists the radiologist, while the general radiology technician operates the C-arm. If the team is inexperienced, they may need to be reminded to position the patient so that both the kidney and the bladder can be visualized fluoroscopically.

Typically a Coons wire is advanced into the bladder through the existing ureteral catheter. If the ureteral catheter had tended to buckle in the tract when it was originally placed, an Amplatz super-staff wire may be a better choice. The catheter is removed over the wire and a 40-cm 9-Fr dilator and sheath are advanced, about half way down the ureter. After the sheath dilator is removed, the sheath can accommodate a second wire that is advanced to the bladder as a safety wire. A super-stiff is not the best as a safety wire, because of a tendency to retract spontaneously. A Coons or Rosen (Cook Medical, Inc., Bloomington, IN) is a good choice for the safety wire.

The safety wire is secured to the drapes with a hemostat. Over the working wire, a $10-\mathrm{mm}$ high-pressure balloon is advanced until its tip is at about the depth at which the collecting system was punctured; oblique fluoroscopy helps judge the proper depth. A 30-Fr sheath is back-loaded on the shaft of the balloon catheter. The balloon is inflated to whatever pressure is necessary to make its "waist" disappear and the tract dilated. The sheath is advanced over the inflated balloon, until their tips are superimposed. The NephroMax
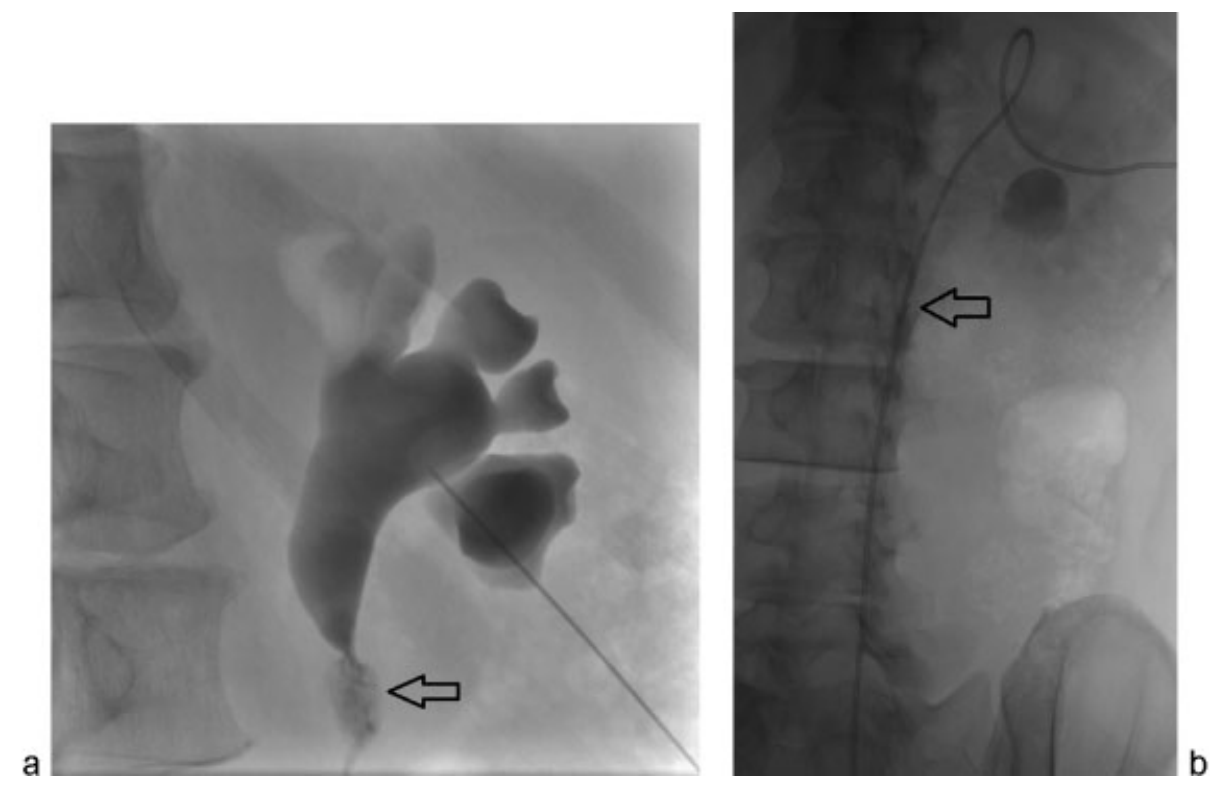

Fig. 11 (a) Nephrostogram demonstrating a large stone in the proximal ureter (arrow). (b) A hydrophilic catheter (arrow) has been advanced past the stone (stone is superimposed upon the inferior corner of L2). 

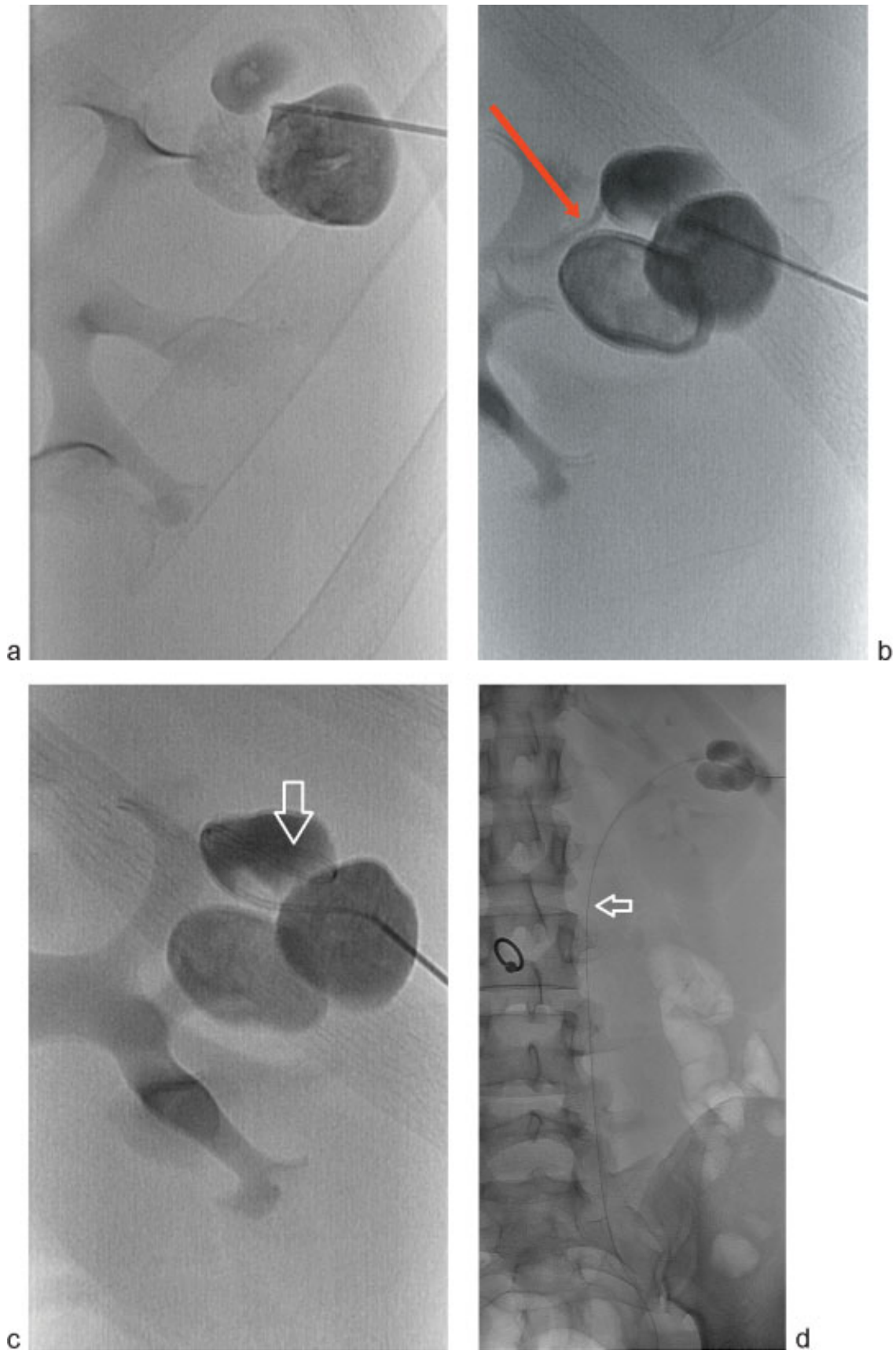

Fig. 12 Stones in a calyceal diverticulum. (a) Direct puncture of the diverticulum is visualized. (b) Arrow demonstrates transient opacification of the communication between the diverticulum and the collecting system. (c) Demonstration of how the guidewire will tend to curl with the diverticulum (white arrow). Persistent gentle probing with the wire will usually be successful. (d) Final image demonstrating catheter placement into the distal ureter (arrow).

High Pressure Nephrostomy Balloon Catheter Kit (Boston Scientific, Natick, MA) works well for most tract dilations.

If the approach is through an empty calyx, and its infundibulum is wide, it is desirable to advance the inflated balloon and sheath deeper into the collecting system, either all the way to the pelvis or wherever the stone is. Advancing the sheath more deeply seals the parenchyma from the collecting system more effectively, allowing less blood to enter the collecting system and thereupon improving the urologist's visualization. One of the difficulties of an approach directly onto a stone is that at first the tip of the sheath lies in the parenchyma, and there is no way to tamponade bleeding with the sheath. In this instance, the urologist's visualization will be compromised until he/she can remove enough stone to advance the sheath over the scope into the collecting system, and thereby tamponading the tract ( $\mathbf{F i g . 1 3}$ ).

Rarely, a kidney may be so deep that a standard sheath is not long enough to extend from the skin to the collecting system. In these cases, one option is to place sutures in the sheath near its superficial edge, and advance it beyond sight 


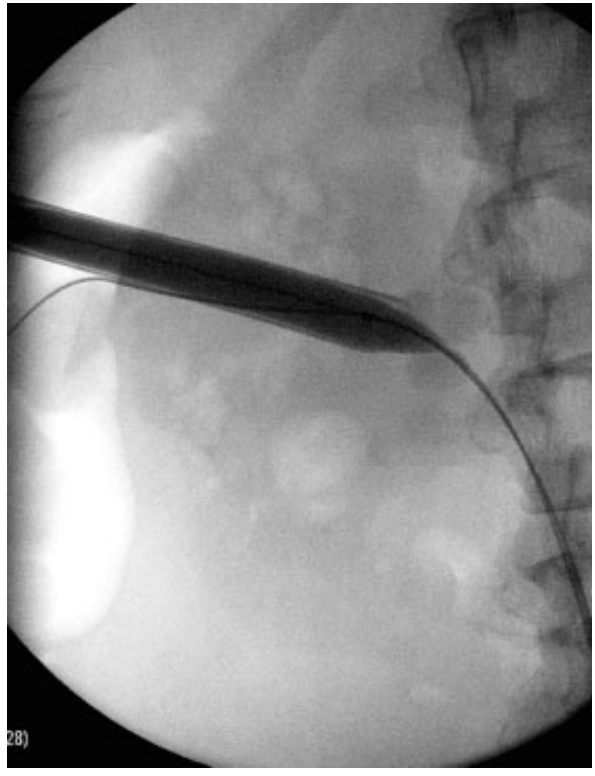

Fig. 13 Image demonstrating the sheath tip being placed over the balloon all the way to the stones. This maneuver should only be performed if the infundibulum is not too narrow.

into the tract. It is vital that the sheath tamponades the tract through the renal parenchyma, both to reduce blood loss and to optimize the urologist's ability to see through the scope.

\section{Confirming Complete Stone Removal}

In the author's practice, as soon as the urologist observes the stone at the tip of the nephroscope, the interventionalist leaves the OR. Most often he or she is not called back, and the urologist carries out the rest of the following steps. Sometimes there is some significant difficulty (for instance, the need for direct intraoperative creation of a new access for an otherwise inaccessible remaining midpole stone) or uncertainty, and the interventionalist is called back to the OR.

Regardless of who remains in the OR, at some point in every case it is time to check to see whether or not there is any stone remaining. Unless the stones were composed of uric acid, which can be suspected from the preprocedure CT, fluoroscopy is the best tool to detect residual stone. It helps rock the sheath tip superiorly and inferiorly while watching under fluoroscopy. The observer should be particularly alert for stone superimposed upon the sheath-the additional density can be very subtle.

A completion nephrostogram does not hurt, but does not usually add much to the fluoroscopic survey; there are usually clots that create filling defects that could easily hide residual stone, and extravasation through rents in the collecting system is common, which may also obscure stones.

\section{Should a Nephrostomy Be Left in Place?}

In the past, at the end of each case the author's routine was to position a 26-Fr 5-mL balloon Foley catheter with its tip and balloon in the collecting system. Its purposes were to tampo- nade the tract and to provide drainage. In addition, over the safety wire, a 5-Fr end-hole catheter was advanced to the bladder. Its functions were partly to act as a stent, and to provide emergency re-access, if necessary. Both catheters were sutured to the skin at the puncture site (-Fig. 14).

In recent years, the author has begun placing double-J ureteral stents $(6,7$, and $8 \mathrm{Fr}$, without any discernable advantage between sizes) and "sealing" the tract with Floseal (Baxter Healthcare Corporation, Deerfield, IL). The urologist injects the Floseal as he/she pulls the scope back from the calyceal entry point to the skin. There is some controversy as to whether the Floseal is truly necessary, but the author has not yet been willing to forgo it. ${ }^{14}$ Regardless of whether or not the Floseal is necessary, the author has not had any complications with this method. It has the added benefit of increased patient satisfaction and decreased length of hospital stay.

\section{Transplant Kidneys}

Because of what is at stake in case of a serious complication, working on transplant kidneys is best left to experienced operators (-Fig. 15). While working on a transplant kidney, combining ultrasound (with color Doppler) and fluoroscopic guidance is helpful. Both modalities can be used to ensure that there is no intervening bowel. The primary puncture is made under direct ultrasound guidance. The kidney is not usually very deep, so ultrasound images are usually of good quality, and color Doppler ought to aid in avoiding even the smallest arteries. Once the needle has reached the target calyx, the ultrasound is put aside, contrast injected, and the remainder of the procedure performed under fluoroscopic control.

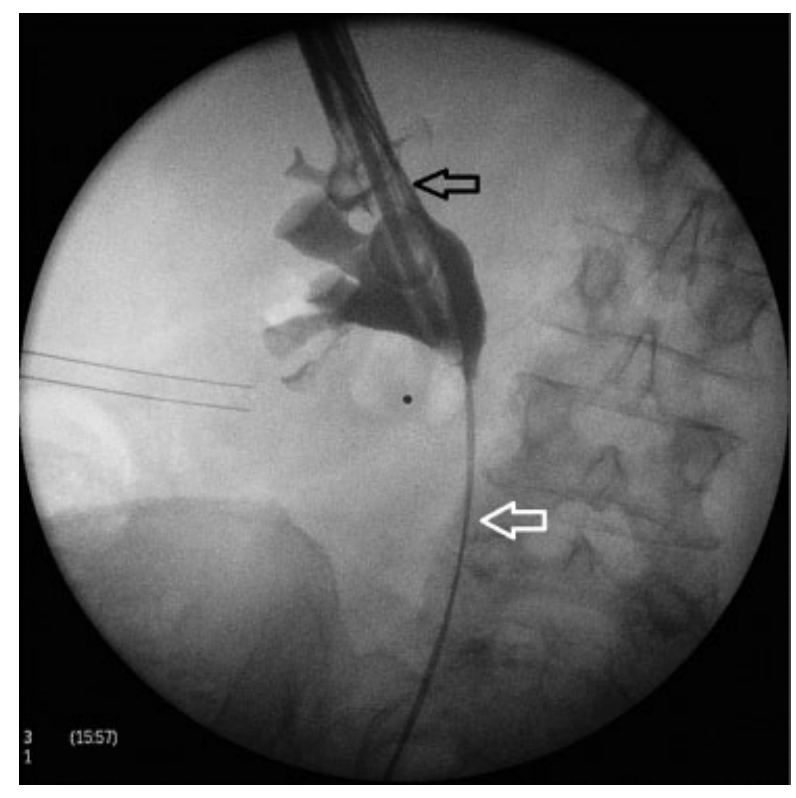

Fig. 14 A 26-Fr Foley catheter with 5-mL balloon has been placed as a nephrostomy catheter (black arrow). A tandem 5-Fr end-hole catheter extends to bladder, acting as a stent (white arrow), and providing reaccess should the Foley be pulled back prematurely. 

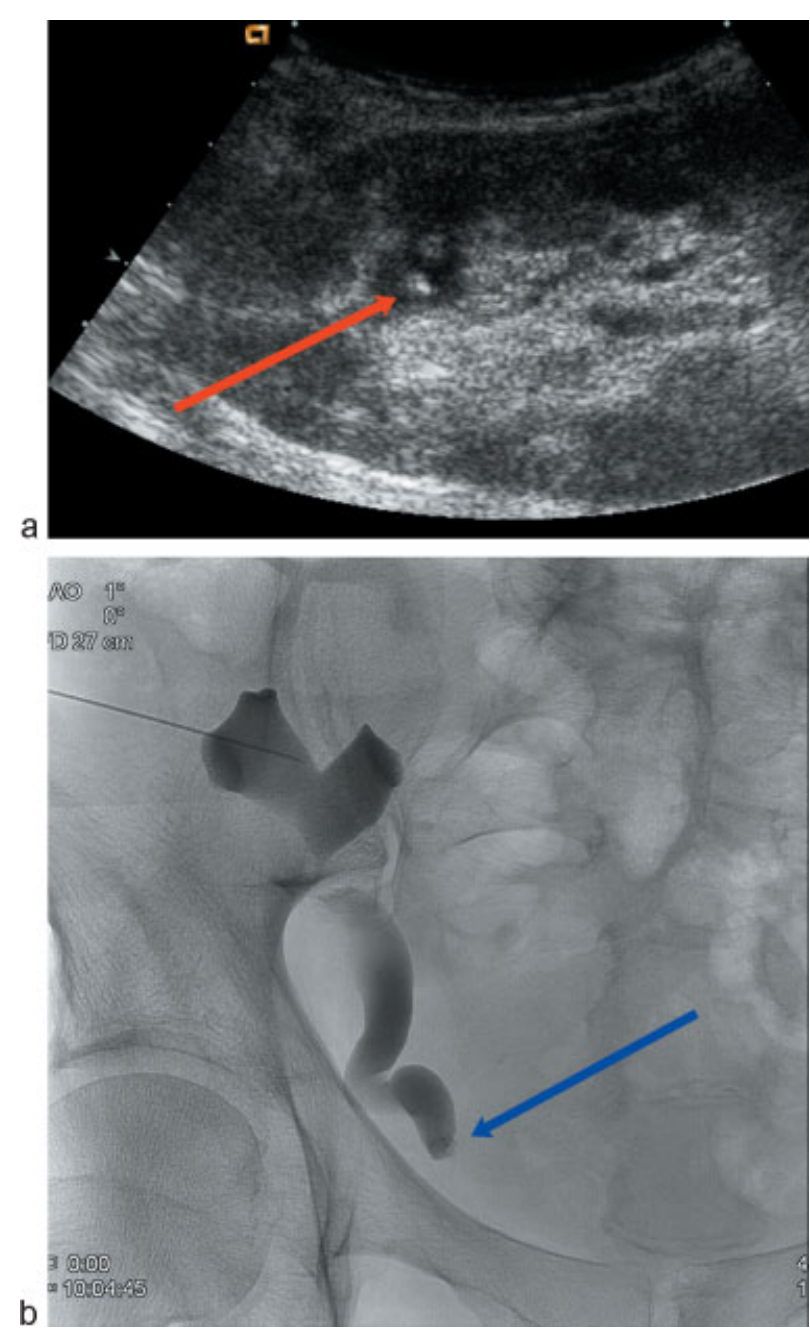

Fig. 15 Access in a transplant kidney. (a) Ultrasound used for calyceal puncture (arrow shows needle tip in the calyx). Note how superficial the kidney lies. (b) Subsequent nephrostogram demonstrates obstructing ureteral stone (arrow).

\section{Preprocedure Items}

This obligatory section has been left until last, although in practice it comes first. There are several significant considerations.

Preprocedural antibiotics are routinely administered. Though the choice of antibiotics is usually left to the discretion of the urologist, preprocedure intravenous antibiotics are administered without exception. Many of the large stones that are treated are "infection stones" known to harbor bacteria. ${ }^{15}$ Sepsis is the most life-threatening and insidious complication associated with these procedures, and unless there is visible hemorrhage or the patient's blood oxygen saturation is low, sepsis should be suspected first if the patient becomes unstable during a case.

Moderate sedation is routinely used for access procedures. If the patient is elderly, has chronic obstructive pulmonary disease, or there is a history of sleep apnea, the amount of medication given should be closely titrated.

These procedures are not usually very painful, but every now and then patients are unusually sensitive to the proce- dure. These patients can feel even the most gentle wire movements. In addition, preexisting spine or shoulder problems may make the position in which these patients are placed unbearable.

Patient's hemoglobin, platelet count, and coagulation parameters are checked routinely. A history of allergy to contrast is problematic; with such a history it is probably best to provide prophylaxis, as almost certainly contrast will reach the bloodstream at some point in the case.

\section{Funding}

No financial support has been received.

\section{Acknowledgments}

The author wishes to acknowledge a profound debt to Dr. Charles J. ("Tunk") Tegtmeyer of the University of Virginia. Tunk's body of work must surely rank him among the great ones of our field. His unwavering intellectual honesty, boundless ingenuity, and unceasing pursuit of excellence were evident to all who worked with him. At the age of 56 years, his untimely death was a tragedy for those who knew him and for the larger world of interventional radiology.

\section{References}

1 Miller RA. Role of endoscopic surgery in management of renal and ureteric calculi: a review. J R Soc Med 1985;78(12): 1034-1038

2 Preminger GM, Assimos DG, Lingeman JE, Nakada SY, Pearle MS, Wolf JS Jr; AUA Nephrolithiasis Guideline Panel). Chapter 1: AUA guideline on management of staghorn calculi: diagnosis and treatment recommendations. J Urol 2005;173(6): 1991-2000

3 Vicentini FC, Gomes CM, Danilovic A, Neto EA, Mazzucchi E, Srougi M. Percutaneous nephrolithotomy: current concepts. Indian J Urol 2009;25(1):4-10

4 Golijanin D, Katz R, Verstandig A, Sasson T, Landau EH, Meretyk S. The supracostal percutaneous nephrostomy for treatment of staghorn and complex kidney stones. J Endourol 1998;12(5): 403-405

5 Munver R, Delvecchio FC, Newman GE, Preminger GM. Critical analysis of supracostal access for percutaneous renal surgery. J Urol 2001;166(4):1242-1246

6 Wong C, Leveillee RJ. Single upper-pole percutaneous access for treatment of $>$ or $=5-\mathrm{cm}$ complex branched staghorn calculi: is shockwave lithotripsy necessary? J Endourol 2002;16(7): 477-481

7 Gupta R, Kumar A, Kapoor R, Srivastava A, Mandhani A. Prospective evaluation of safety and efficacy of the supracostal approach for percutaneous nephrolithotomy. BJU Int 2002;90(9):809-813

8 Rais-Bahrami S, Friedlander JI, Duty BD, Okeke Z, Smith AD. Difficulties with access in percutaneous renal surgery. Ther Adv Urol 2011;3(2):59-68

9 Labate G, Modi P, Timoney A, et al. The percutaneous nephrolithotomy global study: classification of complications. J Endourol 2011;25(8):1275-1280

10 Song SH, Hong B, Park HK, Park T. Paradoxical air embolism during percutaneous nephrolithotomy: a case report. J Korean Med Sci 2007;22(6):1071-1073 
11 Springer RM III. Precise placement of tract anesthesia for percutaneous biliary drainage and nephrostomy. J Vasc Interv Radiol 2000;11(7):938-939

12 Krambeck AE, Lingeman JE. Percutaneous management of caliceal diverticuli. J Endourol 2009;23(10):1723-1729

13 Srivastava A, Chipde SS, Mandhani A, Kapoor R, Ansari MS. Percutaneous management of renal caliceal diverticular stones: Ten-year experience of a tertiary care center with different techniques to deal with diverticula after stone extraction. Indian J Urol 2013;29(4):273-276

14 Shah HN, Hegde S, Shah JN, Mohile PD, Yuvaraja TB, Bansal MB. A prospective, randomized trial evaluating the safety and efficacy of fibrin sealant in tubeless percutaneous nephrolithotomy. J Urol 2006;176(6, Pt 1):2488-2492, discussion 2492-2493

15 Nemoy NJ, Staney TA. Surgical, bacteriological, and biochemical management of "infection stones". JAMA 1971;215(9):1470-1476 